\title{
Title
}

\section{Mutations in the SPTLC1 gene are a cause of juvenile amyotrophic lateral sclerosis that may be amenable to serine supplementation}

\section{Authors}

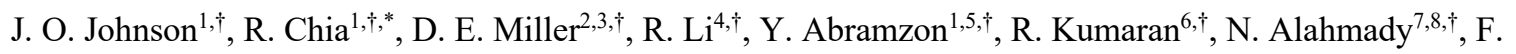
Faghri ${ }^{9,10}$, A. E. Renton ${ }^{1,11,12}$, S. D. Topp ${ }^{7,13}$, H. A. Pliner ${ }^{1}$, J. R. Gibbs ${ }^{14}$, J. Ding ${ }^{14}$, N. Smith ${ }^{6}$, N. Landeck ${ }^{6}$, M. A. Nalls $^{15}$, M. R. Cookson ${ }^{6}$, O. Pletnikova ${ }^{16}$, J. Troncoso ${ }^{16,17}$, S. W. Scholz ${ }^{18,17}$, M. S. Sabir ${ }^{18}$, S. Ahmed ${ }^{18}$, C. L. Dalgard $^{19,20}$, C. Troakes ${ }^{7}$, A. R. Jones ${ }^{7}$, A. Shatunov ${ }^{7}$, A. Iacoangeli ${ }^{7}$, A. Al Khleifat ${ }^{7}$, N. Ticozzi ${ }^{11,22}$, V. Silani ${ }^{11,22}$, C. Gellera $^{23}$, I. P. Blair ${ }^{24}$, C. Dobson-Stone ${ }^{25,26}$, J. B. Kwok ${ }^{25,26}$, B. K. England ${ }^{27}$, E. S. Bonkowski ${ }^{3}$, The International ALS Genomics Consortium $^{28}$, The ITALSGEN Consortium ${ }^{29}$, The FALS Sequencing Consortium ${ }^{30}$, The American Genome Center ${ }^{31}$, P. J. Tienari ${ }^{32}$, D. J. Stone ${ }^{33,}$, K. E. Morrison ${ }^{34}$, P. J. Shaw ${ }^{35}$, A. Al-Chalabi ${ }^{7}$, R. H. Brown, Jr ${ }^{36}$, M. Brunetti $^{37}$, A. Calvo ${ }^{38}$, G. Mora ${ }^{39}$, H. Al-Saif ${ }^{40}$, M. Gotkine ${ }^{41}$, F. Leigh ${ }^{42}$, I. J. Chang 2 , S. J. Perlman ${ }^{42}$, I. Glass ${ }^{2,3}$, C. E. Shaw ${ }^{7,13}$, J. E. Landers ${ }^{36}$, A. Chiò ${ }^{43,44, \uparrow}$, T. O. Crawford ${ }^{45, \uparrow}$, B. N. Smith ${ }^{7, \dagger}$, B. J. Traynor ${ }^{1,17, \dagger}$

\section{Affiliations}

1. Neuromuscular Diseases Research Section, Laboratory of Neurogenetics, National Institute on Aging, National Institutes of Health, Bethesda, MD 20892, USA.

2. Department of Medicine, Division of Medical Genetics, University of Washington, Seattle, WA 98195, USA.

3. Department of Pediatrics, Division of Genetic Medicine, University of Washington, Seattle, WA and Seattle Children's Hospital, Seattle, WA 98105, USA.

4. Department of Pediatrics, Children's Hospital of Richmond at VCU, Richmond, VA 23298, USA.

5. Sobell Department of Motor Neuroscience and Movement Disorders, University College London, Institute of Neurology, London, UK.

6. Cell Biology and Gene Expression Section, Laboratory of Neurogenetics, National Institute on Aging, National Institutes of Health, Bethesda, MD 20892, USA.

7. Maurice Wohl Clinical Neuroscience Institute, Institute of Psychiatry, Psychology and Neuroscience, King's College London, 125 Coldharbour Lane, Camberwell, SE5 9NU, London, UK.

8. Department of Biology, Imam Abdulrahman bin Faisal University, Dammam, Saudi Arabia. 
9. Molecular Genetics Section, Laboratory of Neurogenetics, National Institute on Aging, National Institutes of Health, Bethesda, MD 20892, USA.

10. Department of Computer Science, University of Illinois at Urbana-Champaign, Urbana, IL 61801, USA.

11. Nash Family Department of Neuroscience, Icahn School of Medicine at Mount Sinai, New York, NY 10029, USA.

12. Ronald M. Loeb Center for Alzheimer's Disease, Icahn School of Medicine at Mount Sinai, New York, NY 10029, USA.

13. UK Dementia Research Institute at King's College London, London, UK.

14. Computational Biology Core, Laboratory of Neurogenetics, National Institute on Aging, National Institutes of Health, Bethesda, MD 20892, USA.

15. Data Tecnica International, Glen Echo, MD 20812, USA.

16. Department of Pathology, Johns Hopkins University School of Medicine, Baltimore, MD 21205, USA.

17. Department of Neurology, Johns Hopkins University, Baltimore, MD 21287, USA.

18. Neurodegenerative Diseases Research Unit, Laboratory of Neurogenetics, National Institute of Neurological Disorders and Stroke, National Institutes of Health, Bethesda, MD 20892, USA.

19. Department of Anatomy, Physiology \& Genetics, Uniformed Services University of the Health Sciences, Bethesda, MD 20814, USA.

20. The American Genome Center, Collaborative Health Initiative Research Program, Uniformed Services University of the Health Sciences, Bethesda, MD 20814, USA.

21. Istituto Auxologico Italiano, IRCCS, Department of Neurology-Stroke Unit and Laboratory of Neuroscience, Milan 20145, Italy.

22. Department of Pathophysiology and Transplantation, "Dino Ferrari" Center, Università degli Studi di Milano, Milan 20122, Italy.

23. Unit of Genetics of Neurodegenerative and Metabolic Diseases, Fondazione IRCCS Istituto Neurologico 'Carlo Besta', Milan 20133, Italy.

24. Centre for Motor Neuron Disease Research, Department of Biomedical Sciences, Faculty of Medicine and Health Sciences, Macquarie University, Sydney, New South Wales, Australia.

25. The University of Sydney, Brain and Mind Centre and Central Clinical School, Faculty of Medicine and Health, Camperdown, New South Wales, Australia.

26. School of Medical Sciences, University of New South Wales, Kensington, New South Wales, Australia. 
27. Human Genetics Branch, National Institute of Mental Health, National Institutes of Health, Bethesda, MD 20892, USA.

28. The International ALS Genomics Consortium (see Supplementary Materials for full member list and affiliations).

29. The ITALSGEN Consortium (see Supplementary Materials for full member list and affiliations).

30. The FALS Sequencing Consortium (see Supplementary Materials for full member list and affiliations).

31. The American Genome Center (see Supplementary Materials for full member list and affiliations).

32. Department of Neurology, Helsinki University Hospital and Translational Immunology Programme, Biomedicum, University of Helsinki, Helsinki FIN-02900, Finland.

33. Genetics and Pharmacogenomics, Merck \& Co., Inc., West Point, PA 19486, USA.

34. Faculty of Medicine, Health and Life Sciences, Queen's University Belfast, UK.

35. Sheffield Institute for Translational Neuroscience, Department of Neuroscience, University of Sheffield, Sheffield S10 2HQ, UK.

36. Department of Neurology, University of Massachusetts Medical School, Worcester, MA 01605, USA.

37. Molecular Genetics Unit, Department of Clinical Pathology, A.S.O. O.I.R.M.-S. Anna, 10126 Turin, Italy.

38. 'Rita Levi Montalcini' Department of Neuroscience, University of Turin, Turin, Italy.

39. ALS Center, ICS Maugeri, IRCCS, Milan, Italy.

40. Department of Neurology, Children's Hospital of Richmond at VCU, Richmond, VA 23298, USA.

41. Department of Neurology, The Agnes Ginges Center for Human Neurogenetics, Hadassah-Hebrew University Medical Center, Israel.

42. Department of Neurology, Seattle Children's Hospital, University of Washington, Seattle, WA 98105, USA.

43. ALS Center, 'Rita Levi Montalcini' Department of Neuroscience, University of Turin, Turin, Italy.

44. Neurology 1, AOU Città della Salute e della Scienza, Turin, Italy.

45. Departments of Neurology and Pediatrics, Johns Hopkins University, Baltimore MD 21287, USA.

$\dagger$ These authors contributed equally to this article

$\$$ Current address: Cerevel Therapeutics, Boston, MA 02116, USA.

* Corresponding author. Email: ruth.chia@nih.gov 


\section{Abstract}

Juvenile amyotrophic lateral sclerosis (ALS) is a rare form of childhood motor disorder with a heterogeneous clinical presentation. The underlying causes of this condition are poorly understood, hindering the development of effective therapies. In a whole-exome sequencing trio-family study of three unrelated juvenile patients diagnosed with ALS and failure to thrive, we identified de-novo mutations in SPTLC1 (p.Ala20Ser in two patients and p.Ser331Tyr) not present in their healthy parents or siblings. SPTLC1 encodes a subunit of the serine palmitoyltransferase complex, a key enzyme in sphingolipid biosynthesis. Mutations in this gene are known to cause hereditary sensory autonomic neuropathy, type 1A, with a characteristic increase in plasma levels of neurotoxic deoxymethyl-sphinganine. We found an increase of this metabolite in one of our patients carrying the p.Ala20Ser mutation. Treatment of one of the patients with high dose, oral L-serine led to an increase in body weight, suggesting that serine supplementation may be beneficial among patients carrying mutations in this gene. 


\section{Introduction}

Amyotrophic lateral sclerosis (ALS, OMIM number 105400) is a relatively common neurological disorder characterized by rapidly progressive paralysis leading to death from respiratory failure. The mean age of onset of ALS is the seventh decade of life, with the vast majority of cases occurring after the age of forty $(I)$. In contrast, juvenile ALS (defined as an age of onset less than twenty-five years of age) is a rare form of motor neuron disease (2). These early-onset cases are characterized by relatively slow progression and a variable phenotype that often makes accurate diagnosis challenging (2).

Considerable progress has been made in unravelling the genetic architecture underlying ALS, but much remains to be understood about this condition (3). For example, genome-wide association studies have identified only a handful of loci, many of which have proven difficult to replicate (3). Our lack of knowledge concerning the pathogenesis of ALS hampers efforts to design disease-modifying interventions. Juvenile ALS is thought to be more frequently genetic in origin than the adult-onset forms, and the genetic analysis of these young-onset cases offers an opportunity to identify disease-causing genes (2). By extension, any gene underlying juvenile ALS may also play a role in adultonset cases.

De-novo genetic variants may underlie at least a portion of ALS cases. Such mutations would not be readily detected by genome-wide association studies owing to their recent occurrence and corresponding low frequency within the community. Spontaneously occurring mutations are a well-known cause of neurological conditions, such as neurofibromatosis type 1 and Duchenne muscular dystrophy $(4,5)$. Indeed, de-novo mutations of the familial ALS genes, FUS, SOD1, and $V C P$, have been previously described in sporadic ALS cases (6, 7). Such spontaneous mutations are more likely to present with early-onset disorders due to their impact on fitness (8).

Here, we performed whole-exome sequencing of three patients diagnosed with juvenile ALS and their unaffected parents, to identify the mutations responsible for their disease. None of these cases had a family history of ALS or neuromuscular disorders, suggesting de-novo variations as the underlying genetic mechanism. We also provide preliminary evidence that serine supplementation could revert features of mitochondrial abnormalities in heterologous cell models expressing mutant SPTLC1. Importantly, we demonstrate that oral L-serine significantly improved one of our patient's failure to thrive, which is a promising early clinical proxy following the natural course of the disease. 


\section{Results}

\section{Identification of de novo mutations in SPTLC1 as a cause of juvenile ALS}

We performed whole-exome sequencing of three unrelated patients and their healthy parents who had been diagnosed with juvenile ALS (Figure 1, Table 1). The severe phenotype observed among these patients, together with the lack of a family history of neurological diseases (Figure 2A-C), suggested spontaneous mutations as the underlying genetic defect. The analysis of their genetic data identified de-novo variants in the serine palmitoyltransferase long chain base subunit 1 (SPTLC1) gene that were present in each of the three patients and were absent in their parents

(Figure 2D). Patients 1 and 2 carried the same heterozygous p.Ala20Ser mutation in SPTLC1 due to variation in adjacent nucleotides (chr9:94874844, C >A and chr9:94874843, G>T). Patient 3 carried a p.Ser331Tyr (chr9:92047261G>T) heterozygous mutation in SPTLC1. These SPTLC1 mutations were not present in control subjects or online databases of human polymorphisms $(n=142,489$ individuals $)$. The p.Ser331Tyr amino acid change has been previously implicated in neurological disease (9), whereas the p.Ala20Ser mutations have not been reported previously.

\section{Elevated plasma levels of deoxymethyl-sphinganine in a patient carrying p.Ala20Ser SPTLC1 mutation}

Mutations in SPTLC1 are a known cause of autosomal dominant hereditary sensory and autonomic neuropathy, type 1A (HSAN1, OMIM number 162400) $(10,11)$. The protein encoded by SPTLC1 is an essential subunit of serine palmitoyltransferase (SPT), the enzyme that catalyzes the first and rate-limiting step in the de-novo synthesis of sphingolipids (12). A characteristic feature of SPTLC1 mutations associated with HSAN1 is a shift in substrate specificity of serine palmitoyltransferase to L-alanine and L-glycine, leading to the formation of an atypical class of deoxy-sphingolipids (13). These neurotoxic metabolites accumulate within cells as they cannot be converted to complex sphingolipids nor degraded by the catabolic pathway (13). Abnormally elevated plasma levels of deoxysphingolipids are a known biomarker for pathogenic SPTLC1 mutations in HSAN1 patients (13).

Based on this information, we used mass spectrometry to test for this biomarker in patient 1 carrying the p.Ala20Ser SPTLC1 mutation, and found an abnormally high level of 1-deoxymethyl-sphinganine in her plasma that was not present in her unaffected parents, her unaffected siblings, or control subjects (Figure 2E and Table S2). These findings confirmed that this de-novo mutation had abnormally altered the function of the SPTLC1 protein and was the likely cause of disease in this individual. 


\section{Serine supplementation ameliorates damaging effects caused by mutant p.Ala20Ser}

Using a photometric assay of SPT enzyme activity, we found that the p.Ala20Ser mutant SPTLC1 complex has an altered preference for L-alanine and glycine over the canonical L-serine, compared to the wild-type SPTLC1 complex

(Figure S1). Similar altered kinetics were also observed from cellular-based assays using known HSAN1-

mitochondrial phenotypes (membrane polarization and size) as readouts (14). Measurements were benchmarked against defects found in cells expressing the known HSAN1 mutation p.Cys133Trp that served as an internal positive control. Mitochondrial size and intensity were defective to the same degree in cells expressing p.Ala20Ser and p.Cys133Trp and was reversed to the wild-type phenotype upon the supplementation of serine in culture (Figure S1).

Based on our findings and work published by other groups $(13,15)$, and in light of the poor prognosis observed among juvenile ALS patients, patient 2 was commenced on high-dose oral serine supplementation. She began taking 6 grams per day for five and a half weeks, followed by 9 grams per day for two and a half weeks, and presently is ingesting 10 grams per day. Treatment was well tolerated over six months, and her body weight increased after having stagnated for years (Figure 3). Thus far, we did not observe objective evidence of neurological improvement.

\section{SPTLC1 variants in adult-onset ALS patients are rare}

Having established that mutations in SPTLC1 are a cause of juvenile ALS, we explored the role of mutations in this gene in the pathogenesis of adult-onset ALS. To do this, we evaluated the occurrence of SPTLC1 mutations in a series of 5,607 cases with adult-onset ALS. This screening identified 17 novel SPTLC1 mutations in $19(0 \cdot 34 \%)$ ALS cases that were rare or absent in healthy subjects and were predicted to be damaging (Table S3, Figure S2 and S3). Gene burden testing showed a trend towards significance ( 87 variants in population samples, one-sided Fisher test pvalue using TRAPD software package $=0.0014)$. Due to the late age at presentation of the adult ALS patients, DNA was not available from their parents to determine whether these variants occurred spontaneously. The typical clinical features of ALS were observed among these adult-onset patients, though this subgroup was more likely to report a family history of ALS ( $\mathrm{n}=5$ patients, $26 \cdot 3 \%$, Table 2 ). None of the patients reported sensory or autonomic involvement. The intensity and number of motor neurons staining with SPTLC1 were diminished in autopsy tissue obtained from an ALS patient carrying a p.Arg445Gln mutation in SPTLC1 (Figure S4).

Among the adult-onset ALS cases, we found the p.Ala305Thr variant in SPTLC1 in three patients with familial ALS. These individuals shared a common haplotype extending across the locus, suggesting that they share a common ancestor (Table S5). Though rare ( $\mathrm{n}=2$ out of 135,025 population carried the same SNP), this variant did not segregate within a large, Australian ALS-FTD kindred (Figure S5) (16). Furthermore, a missense mutation in the 
gene CYLD has recently been nominated as the cause of disease in this family (17). Additionally, two siblings in a

UK kindred carried the p.Ala305Thr variant and remained healthy in their seventh decade of life. Overall, these observations point toward p.Ala305Thr being either a non-pathogenic variant or a rare, variably penetrant risk factor.

\section{Discussion}

We provide compelling genetic, biochemical, and cellular data that mutations in SPTLC1 are a cause of juvenile ALS. First, we found three unrelated patients diagnosed with juvenile ALS who carried de-novo mutations in this gene. These variants were not present in our in-house control dataset or online databases of human polymorphisms, indicating they were rare variants in ethnically diverse populations. Two of the patients carried the same alanine to serine amino acid shift at position 20 of the protein which were from different nucleotide changes. Second, mass spectrometry analysis of plasma showed the presence of neurotoxic deoxy-sphingolipid metabolites confirming that the observed mutation alters the function of the encoded enzyme, leading to increased aberrant utilization of alanine and glycine as substrates. This biochemical pattern was consistent with a previously reported disease-causing mechanism in HSAN1 patients (13). Third, we used immunohistochemistry to demonstrate that SPTLC1 is abundantly expressed within the motor neurons of healthy spinal cord tissue.

Though labeled as hereditary sensory and autonomic neuropathy type 1A, the phenotypes associated with mutations in SPTLC1 are known to be varied with patients manifesting various combinations of sensory loss, autonomic dysfunction, and motor weakness (18). Indeed, there is a previous report of a de-novo p.Ser331Phe mutation in SPTLC1 in a young French girl presenting with a similar phenotype to our patients. Her clinical picture consisted of severe restriction of growth, cognitive impairment, amyotrophy, hyperreflexia, vocal cord paralysis, and respiratory failure, though this patient was not formally diagnosed as having juvenile ALS (9). More recently, retinal disease has been reported in patients carrying SPTLC1 mutations (19). This clinical heterogeneity has been linked to the differing effects of each mutation on SPTLC1 enzyme-substrate preference (13), and we observed similar differences in substrate utilization across the variants that we had studied at the enzymatic level (Figure S1).

Perturbed sphingolipid metabolism underlies many neurological disorders, such as Niemann-Pick disease and Gaucher disease (20), and may play a role in the pathogenesis of Alzheimer's disease (21). Sphingolipid metabolism has also been directly implicated in motor neuron degeneration. For example, patients with partial deficiency of hexosaminidase A enzyme activity (also known as GM2 gangliosidosis, a form of sphingolipidosis) may have clinical 
manifestations mimicking ALS (22). Accumulation of ceramides and cholesterol esters also occurs within the spinal cords of ALS patients and a SOD1 transgenic mouse model of ALS (23).

Our data suggest that patients diagnosed with juvenile ALS should be screened for SPTLC1 mutations, especially as dietary supplementation with serine may be beneficial if instituted at an early stage in the disease course (15). Indeed, the treatment of one of our patients with serine resulted in significant weight gain, which was the first time she had gained weight in several years and an initial proof of concept. We did not observe evidence of neurological improvement, though we recognize that prolonged therapy would be required to detect such an effect (24). Serine is a non-essential amino acid that is widely available as a low-cost nutritional supplement. A $10 \%$ serine-enriched diet reduced neurotoxic deoxysphingolipid plasma levels both in transgenic mice expressing the p.Cys133Trp SPTLC1 mutation and in human patients diagnosed with HSAN1 (15). Furthermore, a safety trial involving twenty adult-onset ALS patients demonstrated that high doses of oral serine are well-tolerated and that this polar amino acid is actively transported across the blood-brain barrier (25). Nutritional supplementation has proven to be remarkably effective in other forms of ALS: high dose oral vitamin $\mathrm{B}_{2}$ (riboflavin) slows and even halts neurological progression in BrownVialetto-Van Laere cases, a rare subtype of childhood or young-onset ALS arising from mutations in the riboflavin pathway (26).

Our study has limitations. Our evidence suggesting that mutations in SPTLC1 are a cause of adult-onset ALS is preliminary. Though significance was achieved based on the testing of a single gene, our gene burden testing did not reach genome-wide significance, as the p-value for SPTLC1 in adult-onset ALS cases corrected for multiple testing of 20,000 genes was 1.0. In part, this may be because of the rarity of these mutations and because we could not show segregation of the disease within extended kindreds of the p.Ala305Thr variant. The lack of large pedigrees, due to the poor survival associated with the disease, has hampered the ALS genetics field. Even if mutations in SPTLC1 are confirmed to be a cause of adult-onset ALS by future studies, only a small number of patients diagnosed with ALS are likely to carry pathogenic $S P T L C 1$ mutations. Despite this rarity, the lack of effective treatments for ALS suggests that it may be worthwhile to screen adult ALS patients for SPTLC1 mutations. Serine nutritional supplementation is cheap ( $\$ 500$ per year) and relatively safe (25). In such cases, abnormal deoxysphingolipid plasma metabolites could be used as a diagnostic biomarker to confirm the toxicity of SPTLC1 mutations, and as a marker of target engagement after commencing serine treatment (27).

In conclusion, our data broaden the phenotype associated with mutations in SPTLC1 to include juvenile ALS and implicates sphingolipid metabolism as a pathway in motor neuron disease. Our findings are particularly relevant in 
light of the fact that nutritional supplementation with serine may ameliorate the toxic effect of abnormal sphingolipid metabolites in these patients if instituted at an early stage in the disease. This provides an early opportunity to test the precision medicine approach in an otherwise fatal neurodegenerative disease.

\section{Materials and Methods}

\section{Patients}

Three unrelated patients with neuromuscular symptoms consistent with juvenile ALS took part in the study between March 2016 and March 2020. Table 1 summarizes the clinical features of the three patients. Recruitment information and a detailed description of each case with videos of pertinent neurological findings are available in the appendix.

Patient 1 presented with gradually progressive spastic diplegia and growth retardation at five years of age. By age twenty, she had quadriplegia with marked muscle atrophy and diminished weight, brisk lower limb reflexes, tongue fasciculations and weakness, dysarthria, mild cognitive dysfunction, and respiratory failure requiring tracheostomy and ventilation. Repeated neurophysiological testing did not show evidence of sensory or autonomic dysfunction. She was diagnosed with juvenile ALS based on the revised El Escorial criteria (28).

Patient 2 was a fifteen-year-old, right-handed girl with mixed African American and white race/ethnicity who presented with a six-year history of gradually progressive generalized limb and bulbar weakness. She had a longstanding history of diminished weight of unknown cause, and her school performance began to decline at the age of fifteen. Her neurological examination at presentation revealed a body mass index less than the $1^{\text {st }}$ percentile, exaggerated lumbar lordosis, tongue fasciculations and wasting, generalized muscle atrophy and weakness, brisk asymmetric ankle reflexes, a positive Gower's sign, and normal sensation (Figure 1A-B, Movie S1 and S2). Neurophysiological testing revealed active and chronic denervation without evidence of sensory neuropathy. Decreased sustained attention and impaired executive functioning was evident on neuropsychological evaluation. She was diagnosed with juvenile ALS based on the revised El Escorial criteria (28).

Patient 3 was an eleven-year-old African American girl with a history of failure to gain weight and toe-walking since the age of four. She presented at age ten with a deteriorating gait, hand weakness, right foot paresthesia, dysphagia, and increased sweating. Examination revealed marked atrophy, postural tachycardia, bilateral cataracts, a wasted, fasciculating tongue with an exaggerated jaw jerk, generalized fasciculations and weakness associated with hyperreflexia, and decreased pinprick sensation in a glove-and-stocking distribution (Figure 1C-D, Movie S3). The 
patient walked abnormally due to weakness and bilateral foot drop, and she had a positive Gower's sign.

Neurophysiological examination showed sensorimotor axonal neuropathy, as well as polyphasic potentials on

electromyography. She was diagnosed with a juvenile ALS-plus syndrome due to her prominent motor symptoms and modest sensory-autonomic involvement. The pedigrees of patients 1,2 and 3 are shown in Figure 2A-C.

For mutational screening of SPTLC1 in adult-onset ALS, we used 5,607 DNA samples that were obtained from individuals diagnosed with adult-onset ALS (Table S1). Control data consisted of 5,710 neurologically healthy U.S. individuals who had undergone next-generation sequencing at the Laboratory of Neurogenetics or as part of the Alzheimer's Disease Sequencing Project (ADSP). All subjects provided written informed consent for genetic analysis according to the Declaration of Helsinki, and the Institutional Review Board of the National Institutes of Health approved the study.

\section{Next-generation sequencing in juvenile ALS}

Whole-exome sequencing was performed using 100 base pair, paired-end sequencing on an Illumina sequencer according to the manufacturer's protocol. DNA from patient 1 and her family was sequenced in the Laboratory of Neurogenetics using TruSeq library preparation (version 1.0). DNA from patients 2 and 3 and their families was sequenced at GeneDx using IDT xGen Exome Research Panel (version 1.0) (29).

Data were analyzed to identify de-novo variants present in the affected child, and not present in either parent. As the mutations underlying a rare disease, such as ALS, are unlikely to be present in the general population, variants present in the Genome Aggregation Database (gnomAD, version 2·1), or the Kaviar Genomic Variant database (version September 23, 2015) were excluded. Synonymous, intronic, and intergenic changes were similarly excluded (ANNOVAR, August 11, 2016 version). Paternity and maternity were confirmed using identity-by-descent analysis, and exome data were reviewed to identify mutations in known ALS genes.

\section{SPTLC1 sequencing in adult-onset ALS}

DNA from adult-onset ALS cases were sequenced to identify mutations in the SPTLC1 gene. The sequence data was generated using whole-exome sequencing ( $\mathrm{n}=3,683$ cases) $(30,31)$, whole-genome sequencing $(\mathrm{n}=1,274$ cases), and Sanger sequencing $(\mathrm{n}=650)$ of the fifteen exons and flanking introns of SPTLC1 using the primer sequences listed in Table S4. Variants detected in SPTLC1 based on whole-genome sequence data were confirmed by Sanger sequencing. 
Variants in SPTLC1 were considered to be deleterious if they: (a) were not present in the 4,647 control ADSP subjects; (b) had a frequency less than $3 \cdot 3 \times 10^{-5}$ in online databases of human polymorphisms including the 51,592 European and 8,949 Finnish non-neurological individuals in gnomAD, and the 77,301 samples in Kaviar; ${ }^{1}$ and (c) were designated as 'damaging' according to four out of five prediction algorithms (32); were identified as "stop gain" or "frameshift"; or as splice site mutations with a dbscSNV score higher than $0 \cdot 6$. Gene burden testing of SPTLC1 was performed using publicly available control data (gnomAD and Kaviar) as implemented in the Test Rare vAriants with Public Data (TRAPD) software package (33). This script performs a one-sided Fisher's exact test to determine if there is a higher burden of qualifying variants in cases as compared to controls for a tested gene. The threshold for statistical significance was set at $\mathrm{p} \leq 0.05$.

\section{Measurement of sphingolipid levels}

Sphingolipids were extracted from plasma using methanol extraction (34). Chromatographic separation was performed on an Acquity Ultra Performance Liquid Chromatography system (Waters), and sphingolipids were detected in a Xevo TQ-S triple quadrupole-mass spectrometer (Waters) using the multiple reaction monitoring method. Acquired data were analyzed using MassLynx software (version 4·1). Calibration equations for the sphingolipids were obtained by plotting response against concentration $(\mathrm{ng} / \mathrm{mL})$. The equation showed good linearity over the $1 \mathrm{ng}$ to $3,000 \mathrm{ng}$ range. 


\section{References and Notes}

1. A. Chio, G. Logroscino, B. J. Traynor, J. Collins, J. C. Simeone, L. A. Goldstein, L. A. White, Global epidemiology of amyotrophic lateral sclerosis: a systematic review of the published literature.

Neuroepidemiology. 41, 118-30 (2013).

2. P. Orban, R. S. Devon, M. R. Hayden, B. R. Leavitt, Chapter 15 Juvenile amyotrophic lateral sclerosis. Handb Clin Neurol. 82, 301-12 (2007).

3. R. Chia, A. Chiò, B. J. Traynor, Novel genes associated with amyotrophic lateral sclerosis: diagnostic and clinical implications. Lancet Neurol. 17, 94-102 (2018).

4. M. Littler, N. E. Morton, Segregation analysis of peripheral neurofibromatosis (NF1). J. Med. Genet. 27, 307$310(1990)$.

5. S. Tuffery-Giraud, C. Beroud, F. Leturcq, R. B. Yaou, D. Hamroun, L. Michel-Calemard, M. P. Moizard, R. Bernard, M. Cossee, P. Boisseau, M. Blayau, I. Creveaux, A. Guiochon-Mantel, B. de Martinville, C. Philippe, N. Monnier, E. Bieth, P. Khau Van Kien, F. O. Desmet, V. Humbertclaude, J. C. Kaplan, J. Chelly, M. Claustres, Genotype-phenotype analysis in 2,405 patients with a dystrophinopathy using the UMD-DMD database: a model of nationwide knowledgebase. Hum Mutat. 30, 934-45 (2009).

6. A. Chio, A. Calvo, C. Moglia, I. Ossola, M. Brunetti, L. Sbaiz, S. L. Lai, Y. Abramzon, B. J. Traynor, G. Restagno, Neurobiol Aging, in press, doi:10.1016/j.neurobiolaging.2010.05.016.

7. M. D. Alexander, B. J. Traynor, N. Miller, B. Corr, E. Frost, S. McQuaid, F. M. Brett, A. Green, O. Hardiman, “True” sporadic ALS associated with a novel SOD-1 mutation. Ann. Neurol. 52, 680-683 (2002).

8. R. Acuna-Hidalgo, J. A. Veltman, A. Hoischen, New insights into the generation and role of de novo mutations in health and disease. Genome Biol. 17, 241 (2016).

9. M. Auer-Grumbach, H. Bode, T. R. Pieber, M. Schabhuttl, D. Fischer, R. Seidl, E. Graf, T. Wieland, R. Schuh, G. Vacariu, F. Grill, V. Timmerman, T. M. Strom, T. Hornemann, Mutations at Ser331 in the HSN type I gene SPTLC1 are associated with a distinct syndromic phenotype. Eur J Med Genet. 56, 266-9 (2013).

10. K. Bejaoui, C. Wu, M. D. Scheffler, G. Haan, P. Ashby, L. Wu, P. de Jong, R. H. Brown, SPTLC1 is mutated in hereditary sensory neuropathy, type 1. Nat. Genet. 27, 261-262 (2001). 
11. J. L. Dawkins, D. J. Hulme, S. B. Brahmbhatt, M. Auer-Grumbach, G. A. Nicholson, Mutations in SPTLC1, encoding serine palmitoyltransferase, long chain base subunit-1, cause hereditary sensory neuropathy type I. Nat. Genet. 27, 309-312 (2001).

12. A. H. Merrill, T. H. Stokes, A. Momin, H. Park, B. J. Portz, S. Kelly, E. Wang, M. C. Sullards, M. D. Wang, Sphingolipidomics: a valuable tool for understanding the roles of sphingolipids in biology and disease. J. Lipid Res. 50, S97-S102 (2008).

13. A. Penno, M. M. Reilly, H. Houlden, M. Laura, K. Rentsch, V. Niederkofler, E. T. Stoeckli, G. Nicholson, F. Eichler, R. H. Brown, A. von Eckardstein, T. Hornemann, Hereditary Sensory Neuropathy Type 1 Is Caused by the Accumulation of Two Neurotoxic Sphingolipids. J. Biol. Chem. 285, 11178-11187 (2010).

14. E. R. Wilson, U. Kugathasan, A. Y. Abramov, A. J. Clark, D. L. H. Bennett, M. M. Reilly, L. Greensmith, B. Kalmar, Hereditary sensory neuropathy type 1-associated deoxysphingolipids cause neurotoxicity, acute calcium handling abnormalities and mitochondrial dysfunction in vitro. Neurobiol. Dis. 117, 1-14 (2018).

15. K. Garofalo, A. Penno, B. P. Schmidt, H.-J. Lee, M. P. Frosch, A. von Eckardstein, R. H. Brown, T. Hornemann, F. S. Eichler, Oral 1-serine supplementation reduces production of neurotoxic deoxysphingolipids in mice and humans with hereditary sensory autonomic neuropathy type 1. J. Clin. Invest. 121, 4735-4745 (2011).

16. C. Dobson-Stone, A. A. Luty, E. M. Thompson, P. Blumbergs, W. S. Brooks, C. L. Short, C. D. Field, P. K. Panegyres, J. Hecker, J. A. Solski, I. P. Blair, J. M. Fullerton, G. M. Halliday, P. R. Schofield, J. B. J. Kwok, Frontotemporal dementia-amyotrophic lateral sclerosis syndrome locus on chromosome 16p12.1-q12.2: genetic, clinical and neuropathological analysis. Acta Neuropathol. (Berl.). 125, 523-533 (2013).

17. C. Dobson-Stone, M. Hallupp, H. Shahheydari, A. M. G. Ragagnin, Z. Chatterton, F. Carew-Jones, C. E. Shepherd, H. Stefen, E. Paric, T. Fath, E. M. Thompson, P. Blumbergs, C. L. Short, C. D. Field, P. K. Panegyres, J. Hecker, G. Nicholson, A. D. Shaw, J. M. Fullerton, A. A. Luty, P. R. Schofield, W. S. Brooks, N. Rajan, M. F. Bennett, M. Bahlo, Shankaracharya, J. E. Landers, O. Piguet, J. R. Hodges, G. M. Halliday, S. D. Topp, B. N. Smith, C. E. Shaw, E. McCann, J. A. Fifita, K. L. Williams, J. D. Atkin, I. P. Blair, J. B. Kwok, CYLD is a causative gene for frontotemporal dementia - amyotrophic lateral sclerosis. Brain. 143, 783-799 (2020). 
18. G. A. Nicholson, in GeneReviews ${ }^{\circledR}$, M. P. Adam, H. H. Ardinger, R. A. Pagon, S. E. Wallace, L. J. Bean, K.

Stephens, A. Amemiya, Eds. (University of Washington, Seattle, Seattle (WA), 1993;

http://www.ncbi.nlm.nih.gov/books/NBK1390/).

19. M. L. Gantner, K. Eade, M. Wallace, M. K. Handzlik, R. Fallon, J. Trombley, R. Bonelli, S. Giles, S. Harkins-

Perry, T. F. C. Heeren, L. Sauer, Y. Ideguchi, M. Baldini, L. Scheppke, M. I. Dorrell, M. Kitano, B. J. Hart, C.

Cai, T. Nagasaki, M. G. Badur, M. Okada, S. M. Woods, C. Egan, M. Gillies, R. Guymer, F. Eichler, M.

Bahlo, M. Fruttiger, R. Allikmets, P. S. Bernstein, C. M. Metallo, M. Friedlander, Serine and Lipid Metabolism in Macular Disease and Peripheral Neuropathy. N Engl J Med (2019), doi:10.1056/NEJMoa1815111.

20. F. Sabourdy, L. Astudillo, C. Colacios, P. Dubot, M. Mrad, B. Ségui, N. Andrieu-Abadie, T. Levade, Monogenic neurological disorders of sphingolipid metabolism. Biochim. Biophys. Acta. 1851, 1040-1051 (2015).

21. N. J. Haughey, V. V. R. Bandaru, M. Bae, M. P. Mattson, Roles for dysfunctional sphingolipid metabolism in Alzheimer's disease neuropathogenesis. Biochim. Biophys. Acta. 1801, 878-886 (2010).

22. H. Mitsumoto, R. J. Sliman, I. A. Schafer, C. S. Sternick, B. Kaufman, A. Wilbourn, S. J. Horwitz, Motor neuron disease and adult hexosaminidase A deficiency in two families: evidence for multisystem degeneration. Ann Neurol. 17, 378-85 (1985).

23. R. G. Cutler, W. A. Pedersen, S. Camandola, J. D. Rothstein, M. P. Mattson, Evidence that accumulation of ceramides and cholesterol esters mediates oxidative stress-induced death of motor neurons in amyotrophic lateral sclerosis. Ann. Neurol. 52, 448-457 (2002).

24. V. Fridman, S. Suriyanarayanan, P. Novak, W. David, E. A. Macklin, D. McKenna-Yasek, K. Walsh, R. AzizBose, A. L. Oaklander, R. Brown, T. Hornemann, F. Eichler, Randomized trial of l-serine in patients with hereditary sensory and autonomic neuropathy type 1. Neurology. 92, e359-e370 (2019).

25. T. D. Levine, R. G. Miller, W. G. Bradley, D. H. Moore, D. S. Saperstein, L. E. Flynn, J. S. Katz, D. A. Forshew, J. S. Metcalf, S. A. Banack, P. A. Cox, Phase I clinical trial of safety of L-serine for ALS patients. Amyotroph. Lateral Scler. Front. Degener. 18, 107-111 (2017). 
26. A. R. Foley, M. P. Menezes, A. Pandraud, M. A. Gonzalez, A. Al-Odaib, A. J. Abrams, K. Sugano, A.

Yonezawa, A. Y. Manzur, J. Burns, I. Hughes, B. G. McCullagh, H. Jungbluth, M. J. Lim, J.-P. Lin, A.

Megarbane, J. A. Urtizberea, A. H. Shah, J. Antony, R. Webster, A. Broomfield, J. Ng, A. A. Mathew, J. J.

O’Byrne, E. Forman, M. Scoto, M. Prasad, K. O’Brien, S. Olpin, M. Oppenheim, I. Hargreaves, J. M. Land, M.

X. Wang, K. Carpenter, R. Horvath, V. Straub, M. Lek, W. Gold, M. O. Farrell, S. Brandner, R. Phadke, K.

Matsubara, M. L. McGarvey, S. S. Scherer, P. S. Baxter, M. D. King, P. Clayton, S. Rahman, M. M. Reilly, R.

A. Ouvrier, J. Christodoulou, S. Züchner, F. Muntoni, H. Houlden, Treatable childhood neuronopathy caused

by mutations in riboflavin transporter RFVT2. Brain J. Neurol. 137, 44-56 (2014).

27. F. Boso, A. Armirotti, F. Taioli, M. Ferrarini, L. Nobbio, T. Cavallaro, G. M. Fabrizi, Deoxysphingolipids as candidate biomarkers for a novel SPTLC1 mutation associated with HSAN-I. Neurol Genet. 5, e365 (2019).

28. B. R. Brooks, R. G. Miller, M. Swash, T. L. Munsat, World Federation of Neurology Research Group on Motor Neuron Diseases, El Escorial revisited: revised criteria for the diagnosis of amyotrophic lateral sclerosis. Amyotroph. Lateral Scler. Mot. Neuron Disord. Off. Publ. World Fed. Neurol. Res. Group Mot. Neuron Dis. 1, 293-299 (2000).

29. K. Retterer, J. Juusola, M. T. Cho, P. Vitazka, F. Millan, F. Gibellini, A. Vertino-Bell, N. Smaoui, J. Neidich, K. G. Monaghan, D. McKnight, R. Bai, S. Suchy, B. Friedman, J. Tahiliani, D. Pineda-Alvarez, G. Richard, T. Brandt, E. Haverfield, W. K. Chung, S. Bale, Clinical application of whole-exome sequencing across clinical indications. Genet Med. 18, 696-704 (2016).

30. Project MinE ALS Sequencing Consortium, Project MinE: study design and pilot analyses of a large-scale whole-genome sequencing study in amyotrophic lateral sclerosis. Eur. J. Hum. Genet. EJHG. 26, 1537-1546 (2018).

31. C. Raczy, R. Petrovski, C. T. Saunders, I. Chorny, S. Kruglyak, E. H. Margulies, H.-Y. Chuang, M. Källberg, S. A. Kumar, A. Liao, K. M. Little, M. P. Strömberg, S. W. Tanner, Isaac: ultra-fast whole-genome secondary analysis on Illumina sequencing platforms. Bioinforma. Oxf. Engl. 29, 2041-2043 (2013).

32. D. Anderson, T. Lassmann, A phenotype centric benchmark of variant prioritisation tools. NPJ Genomic Med. 3, 5 (2018). 
33. M. H. Guo, L. Plummer, Y.-M. Chan, J. N. Hirschhorn, M. F. Lippincott, Burden Testing of Rare Variants Identified through Exome Sequencing via Publicly Available Control Data. Am. J. Hum. Genet. 103, 522-534 (2018).

34. A. H. Merrill, M. C. Sullards, J. C. Allegood, S. Kelly, E. Wang, Sphingolipidomics: high-throughput, structure-specific, and quantitative analysis of sphingolipids by liquid chromatography tandem mass spectrometry. Methods San Diego Calif. 36, 207-224 (2005).

35. C. B. Brady, K. T. Trevor, T. D. Stein, E. Y. Deykin, S. D. Perkins, J. G. Averill, L. Guilderson, A. C. Mckee, S. W. Renner, N. W. Kowall, The Department of Veterans Affairs Biorepository Brain Bank: A national resource for amyotrophic lateral sclerosis research. Amyotroph. Lateral Scler. Front. Degener. 14, 591-597 (2013).

36. N. Pilpel, N. Landeck, M. Klugmann, P. H. Seeburg, M. K. Schwarz, Rapid, reproducible transduction of select forebrain regions by targeted recombinant virus injection into the neonatal mouse brain. J Neurosci Methods.

182, 55-63 (2009).

37. R. Chia, S. Haddock, A. Beilina, I. N. Rudenko, A. Mamais, A. Kaganovich, Y. Li, R. Kumaran, M. A. Nalls, M. R. Cookson, Phosphorylation of LRRK2 by casein kinase 1alpha regulates trans-Golgi clustering via differential interaction with ARHGEF7. Nat Commun. 5, 5827 (2014).

38. M. F. Rutti, S. Richard, A. Penno, A. von Eckardstein, T. Hornemann, An improved method to determine serine palmitoyltransferase activity. J Lipid Res. 50, 1237-44 (2009). 
bioRxiv preprint doi: https://doi.org/10.1101/770339; this version posted June 29, 2020. The copyright holder for this preprint (which was not

certified by peer review) is the author/funder. This article is a US Government work. It is not subject to copyright under 17 USC 105 and is also made available for use under a CCO license.

\section{Acknowledgments}

General: We thank the patients and their families for their participation in our research. We also thank Kirsty McWalter and GeneDx for their assistance. This study used DNA samples, genotype data, and clinical data from the NINDS Repository at Coriell, the New York Brain Bank-The Taub Institute, Columbia University, Department of Veterans Affairs Biorepository Brain Bank (grant \#BX002466), the Baltimore Longitudinal Study of Aging, the Johns Hopkins University Alzheimer's Disease Research Center (NIH grant P50AG05146), the NICHD Brain and Tissue Bank for Developmental Disorders at the University of Maryland, the MRC London Neurodegenerative Diseases Brain Bank, Kings College London, Denmark Hill, London, UK, and the Alzheimer's Disease Sequencing Project (www.niagads.org/adsp for more details). Samples used in this research were obtained from the U.K. MND Collections funded by the MND Association and the Wellcome Trust. We thank Peter R. Schofield, William S. Brooks, Elizabeth M. Thompson, Peter Blumbergs, Cathy L. Short, Colin D. Field, Peter K. Panegyres, and Jane Hecker for their assistance in collecting samples from the Australian kindred.

Funding: This work was supported in part by the Intramural Research Programs of the NIH, National Institute on Aging (Z01-AG000949-02), and NINDS (ZIA-NS03154). The work was also funded by the Packard Center for ALS Research at Hopkins, the ALS Association, the Muscular Dystrophy Association, the Italian Ministry of Health (RF2016-02362405), the Italian Ministry of Education, University and Research (Progetti di Ricerca di Rilevante Interesse Nazionale, PRIN, grant n. 2017SNW5MB), the Joint Programme - Neurodegenerative Disease Research (JPND, Brain-Mend projects) granted by Italian Ministry of Education, University and Research, and by the European Community's Health Seventh Framework Programme (FP7/2007-2013, grant agreements no. 259867 and 278611), by the National Institute of Neurological Disorders and Stroke (NIH grant number R35 NS097261), and by the Collaborative Health Initiative Research Program. This study was performed under the Department of Excellence grant of the Italian Ministry of Education, University and Research to the 'Rita Levi Montalcini' Department of Neuroscience, University of Torino, Italy. Additional funding was also provided by the Motor Neurone Disease Association (MNDA), the Medical Research Council, the Medical Research Foundation (MRF), the Van Geest Foundation, The Psychiatry Research Trust of the Institute of Psychiatry, Guy's and St. Thomas' Charity and the Noreen Murray Foundation, the Sigrid Juselius Foundation, the UK Dementia Research Institute, the National Health and Medical Research Council of Australia (1095215, 1092023), and through the following funding organisations under the aegis of JPND: United Kingdom, Medical Research Council (MR/L501529/1; MR/R024804/1). This work was supported by the UK Dementia Research Institute which is funded by the Medical Research Council, 
Alzheimer's Society and Alzheimer's Research UK. Ammar Al-Chalabi is supported by the National Institute for Health Research (NIHR) Maudsley Biomedical Research Centre. Pamela Shaw is supported as an NIHR Senior Investigator and by the Sheffield NIHR Biomedical research Centre. Carol Dobson-Stone is supported by the Australian National Health and Medical Research Council (NHMRC) Boosting Dementia Research Leadership Fellowship 1138223 and by the University of Sydney. John B. Kwok is supported by NHMRC Dementia Research Team Grant 1095127.

The Alzheimer's Disease Sequencing Project (ADSP) is comprised of two Alzheimer's Disease (AD) genetics consortia and three National Human Genome Research Institute (NHGRI) funded Large Scale Sequencing and Analysis Centers (LSAC). The two AD genetics consortia are the Alzheimer's Disease Genetics Consortium (ADGC) funded by NIA (U01 AG032984), and the Cohorts for Heart and Aging Research in Genomic Epidemiology (CHARGE) funded by NIA (R01 AG033193), the National Heart, Lung, and Blood Institute (NHLBI), other National Institute of Health (NIH) institutes and other foreign governmental and non-governmental organizations. The Discovery Phase analysis of sequence data is supported through UF1AG047133 (to Drs. Schellenberg, Farrer, Pericak-Vance, Mayeux, and Haines); U01AG049505 to Dr. Seshadri; U01AG049506 to Dr. Boerwinkle; U01AG049507 to Dr. Wijsman; and U01AG049508 to Dr. Goate and the Discovery Extension Phase analysis is supported through U01AG052411 to Dr. Goate, U01AG052410 to Dr. Pericak-Vance and U01 AG052409 to Drs. Seshadri and Fornage. Data generation and harmonization in the Follow-up Phases is supported by U54AG052427 (to Drs. Schellenberg and Wang). The ADGC cohorts include: Adult Changes in Thought (ACT), the Alzheimer's Disease Centers (ADC), the Chicago Health and Aging Project (CHAP), the Memory and Aging Project (MAP), Mayo Clinic (MAYO), Mayo Parkinson's Disease controls, University of Miami, the Multi-Institutional Research in Alzheimer's Genetic Epidemiology Study (MIRAGE), the National Cell Repository for Alzheimer's Disease (NCRAD), the National Institute on Aging Late Onset Alzheimer's Disease Family Study (NIA-LOAD), the Religious Orders Study (ROS), the Texas Alzheimer's Research and Care Consortium (TARC), Vanderbilt University/Case Western Reserve University (VAN/CWRU), the Washington Heights-Inwood Columbia Aging Project (WHICAP) and the Washington University Sequencing Project (WUSP), the Columbia University HispanicEstudio Familiar de Influencia Genetica de Alzheimer (EFIGA), the University of Toronto (UT), and Genetic Differences (GD). The CHARGE cohorts are supported in part by National Heart, Lung, and Blood Institute (NHLBI) infrastructure grant HL105756 (Psaty), RC2HL102419 (Boerwinkle) and the neurology working group is supported by the National Institute on Aging (NIA) R01 grant AG033193. The CHARGE cohorts participating in the ADSP include the following: Austrian Stroke Prevention Study (ASPS), ASPS-Family study, and the Prospective Dementia Registry-Austria (ASPS/PRODEM-Aus), the Atherosclerosis Risk in Communities (ARIC) Study, the 
bioRxiv preprint doi: https://doi.org/10.1101/770339; this version posted June 29, 2020. The copyright holder for this preprint (which was not

certified by peer review) is the author/funder. This article is a US Government work. It is not subject to copyright under 17 USC 105 and is also made available for use under a CCO license.

Cardiovascular Health Study (CHS), the Erasmus Rucphen Family Study (ERF), the Framingham Heart Study (FHS), and the Rotterdam Study (RS). ASPS is funded by the Austrian Science Fond (FWF) grant number P20545-P05 and P13180 and the Medical University of Graz. The ASPS-Fam is funded by the Austrian Science Fund (FWF) project I904), the EU Joint Programme - Neurodegenerative Disease Research (JPND) in frame of the BRIDGET project (Austria, Ministry of Science) and the Medical University of Graz and the Steiermärkische Krankenanstalten Gesellschaft. PRODEM-Austria is supported by the Austrian Research Promotion agency (FFG) (Project No. 827462) and by the Austrian National Bank (Anniversary Fund, project 15435. ARIC research is carried out as a collaborative study supported by NHLBI contracts (HHSN268201100005C, HHSN268201100006C, HHSN268201100007C, HHSN268201100008C, HHSN268201100009C, HHSN268201100010C, HHSN268201100011C, and HHSN268201100012C). Neurocognitive data in ARIC is collected by U01 2U01HL096812, 2U01HL096814, 2U01HL096899, 2U01HL096902, 2U01HL096917 from the NIH (NHLBI, NINDS, NIA and NIDCD), and with previous brain MRI examinations funded by R01-HL70825 from the NHLBI. CHS research was supported by contracts HHSN268201200036C, HHSN268200800007C, N01HC55222, N01HC85079, N01HC85080, N01HC85081, N01HC85082, N01HC85083, N01HC85086, and grants U01HL080295 and U01HL130114 from the NHLBI with additional contribution from the National Institute of Neurological Disorders and Stroke (NINDS). Additional support was provided by R01AG023629, R01AG15928, and R01AG20098 from the NIA. FHS research is supported by NHLBI contracts N01-HC-25195 and HHSN268201500001I. This study was also supported by additional grants from the NIA (R01s AG054076, AG049607 and AG033040 and NINDS (R01 NS017950). The ERF study as a part of EUROSPAN (European Special Populations Research Network) was supported by European Commission FP6 STRP grant number 018947 (LSHG-CT-2006-01947) and also received funding from the European Community's Seventh Framework Programme (FP7/2007-2013)/grant agreement HEALTH-F4-2007-201413 by the European Commission under the programme "Quality of Life and Management of the Living Resources" of 5th Framework Programme (no. QLG2-CT-200201254). High-throughput analysis of the ERF data was supported by a joint grant from the Netherlands Organization for Scientific Research and the Russian Foundation for Basic Research (NWO-RFBR 047.017.043). The Rotterdam Study is funded by Erasmus Medical Center and Erasmus University, Rotterdam, the Netherlands Organization for Health Research and Development (ZonMw), the Research Institute for Diseases in the Elderly (RIDE), the Ministry of Education, Culture and Science, the Ministry for Health, Welfare and Sports, the European Commission (DG XII), and the municipality of Rotterdam. Genetic data sets are also supported by the Netherlands Organization of Scientific Research NWO Investments (175.010.2005.011, 911-03-012), the Genetic Laboratory of the Department of Internal Medicine, Erasmus MC, the Research Institute for Diseases in the Elderly (014-93-015; RIDE2), and the Netherlands 
Genomics Initiative (NGI)/Netherlands Organization for Scientific Research (NWO) Netherlands Consortium for Healthy Aging (NCHA), project 050-060-810. All studies are grateful to their participants, faculty and staff. The content of these manuscripts is solely the responsibility of the authors and does not necessarily represent the official views of the National Institutes of Health or the U.S. Department of Health and Human Services. The four LSACs are: the Human Genome Sequencing Center at the Baylor College of Medicine (U54 HG003273), the Broad Institute Genome Center (U54HG003067), The American Genome Center at the Uniformed Services University of the Health Sciences (U01AG057659), and the Washington University Genome Institute (U54HG003079). Biological samples and associated phenotypic data used in primary data analyses were stored at Study Investigators institutions, and at the National Cell Repository for Alzheimer's Disease (NCRAD, U24AG021886) at Indiana University funded by NIA. Associated Phenotypic Data used in primary and secondary data analyses were provided by Study Investigators, the NIA funded Alzheimer's Disease Centers (ADCs), and the National Alzheimer's Coordinating Center (NACC, U01AG016976) and the National Institute on Aging Genetics of Alzheimer's Disease Data Storage Site (NIAGADS, U24AG041689) at the University of Pennsylvania, funded by NIA, and at the Database for Genotypes and Phenotypes (dbGaP, phs000572) funded by NIH. This research was supported in part by the Intramural Research Program of the National Institutes of Health, National Library of Medicine. Contributors to the Genetic Analysis Data included Study Investigators on projects that were individually funded by NIA and other NIH institutes, and by private U.S. organizations, or foreign governmental or nongovernmental organizations.

VS, NT, and CG were financially supported by the Italian Ministry of Health (Grant RF-201302355764).

Author contributions: RC, RK, JEL, ACh, TC, BNS, and BJT designed the study, wrote the report, did the literature search, and drew the figures. OP, JT, DEM, The International ALS Genomics Consortium; The ITALSGEN Consortium; the FALS Consortium, Project MinE, IB, CD-S, JBK, RHB, ACa, GM, ACh, TC, CES, MG, BNS, and BJT obtained samples and clinical data from patients. JOJ, RC, RK, YA, FF, AER, HAP, SDT, NA, JRG, JD, MAN, CD-S, MN, CLD, SWS, MSS, SA, IG, FL, JEL, BNS, ACh, IJC, TC, and BJT performed experiments and the data analysis.

Competing interests: PJT and BJT hold the US, Canadian and European patents on the clinical testing and therapeutic intervention for the hexanucleotide repeat expansion in C9orf72. ACh serves on scientific advisory boards for Biogen Idec, Cytokinetics, Italfarmaco, and Neuraltus. AA-C reports consultancies for Biogen Idec, Cytokinetics Inc, OrionPharma, Chronos Therapeutics, and Mitsubishi-Tanabe Pharma. The other authors report no conflicts of interest. 
bioRxiv preprint doi: https://doi.org/10.1101/770339; this version posted June 29, 2020. The copyright holder for this preprint (which was not certified by peer review) is the author/funder. This article is a US Government work. It is not subject to copyright under 17 USC 105 and is also made available for use under a CCO license.

\section{Figures and Tables}
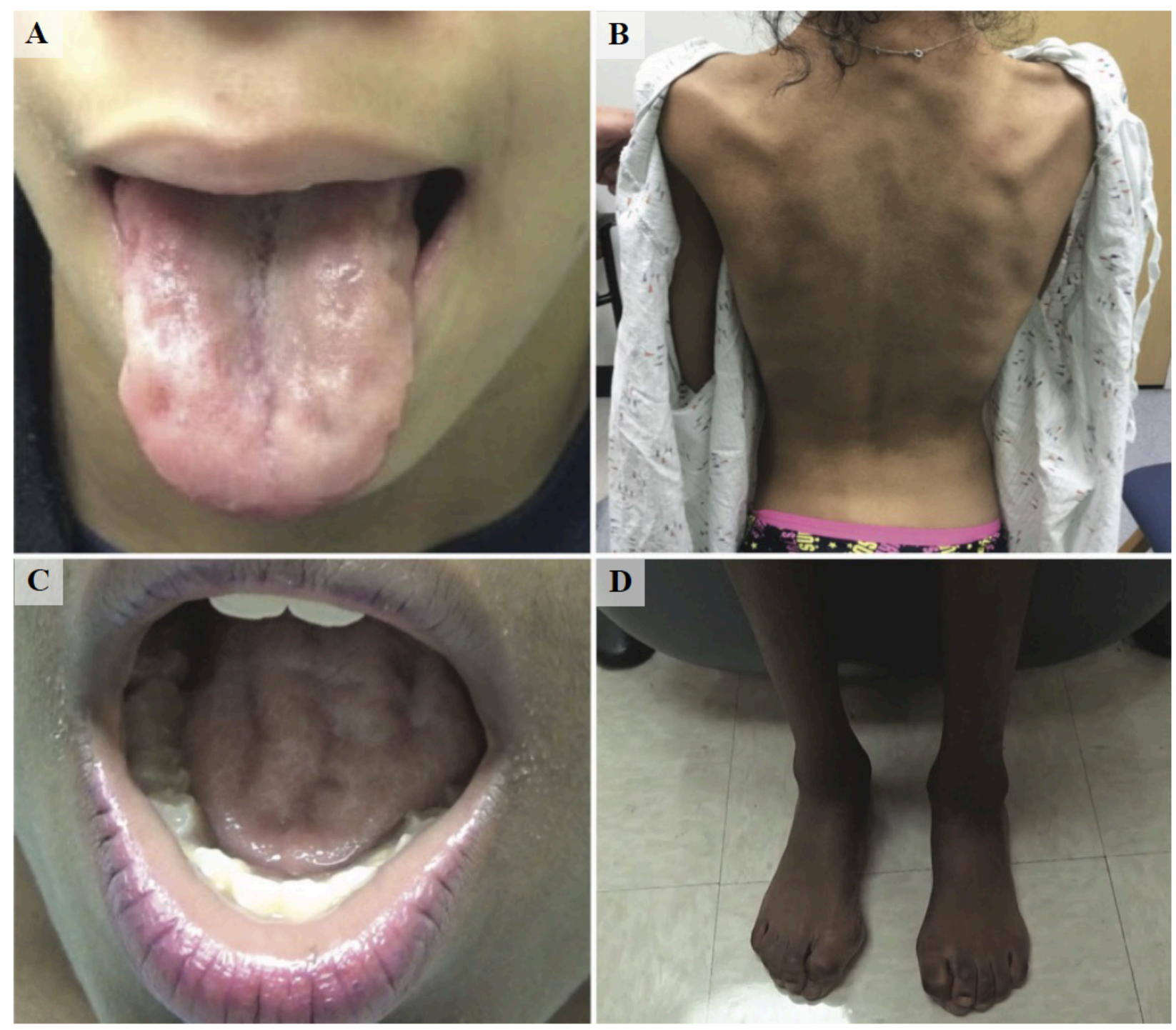

Figure 1. Clinical features of the patients diagnosed with juvenile ALS.

(A \& B) Tongue wasting and scapular winging in patient 2 carrying the p.Ala20Ser SPTLC1 mutation. (C \&D)

Tongue wasting and muscle atrophy of the lower limbs in patient 3 carrying the p.Ser331Tyr SPTLC1 mutation. Note the hammertoe deformities of both feet. 



Figure 2. De-novo mutations of SPTLC1 in patients diagnosed with juvenile ALS.

(A-C) Pedigree of three patients diagnosed with juvenile ALS. The mutant alleles in SPTLC1 are indicated by $m t$, whereas wild-type alleles are indicated by $w t$. The arrows indicate the probands. (D) Distribution of SPTLC1 mutations detected in patients diagnosed with juvenile ALS. Mutations identified in the three juvenile ALS cases are noted in red, and mutations previously described to cause HSAN1 are shown in green. (E) Plasma analysis of the proband and healthy controls (unaffected mother, unaffected brother, and two unrelated healthy individuals). Proband had $\sim 1.8$ times higher levels of 1-deoxymethyl-sphinganine and 1-deoxymethyl-sphingosine compared to the healthy controls. The utilization of L-alanine by the serine palmitoyltransferase complex produces 1-deoxysphinganine (m18:0) and 1-deoxysphingosine (m18:1), whereas the use of L-glycine yields 1-deoxymethyl-sphinganine (m17:0) and 1-deoxymethyl-sphingosine (m17:1). Collectively, these non-canonical metabolites are called deoxysphingoid bases. 


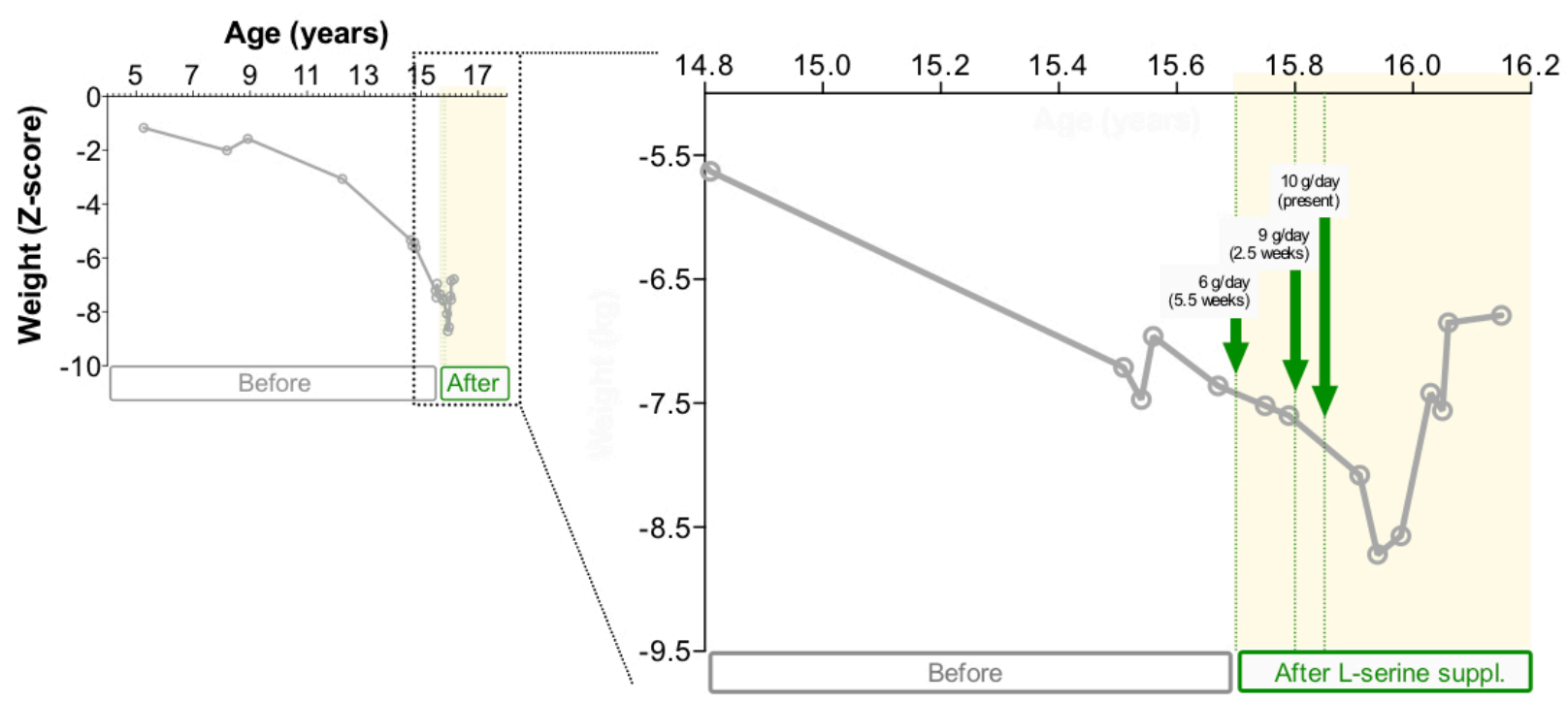

Figure 3. Body mass index progression of patient 2 on high-dose oral serine supplementation.

The weight profile of patient 2 indicated a steady loss in weight until she was started on high-dose oral serine supplementation at the age of 15.7 years. Weights are presented as Body mass index (BMI) z-scores that are measures of relative weight adjusted for child age and sex. Higher values represent higher BMI. The vertical dashed lines indicate dose increases. Treatment was well tolerated over six months, and her weight increased, surpassing her maximum recorded weight. 
Table 1. Clinical features of patients diagnosed with juvenile ALS and carrying de-novo mutations in SPTLC1

\begin{tabular}{|c|c|c|c|}
\hline Clinical features & Patient 1 & Patient 2 & Patient 3 \\
\hline Gene change & p.Ala20Ser & p.Ala20Ser* & p.Ser331Tyr \\
\hline Age at onset & $5 \mathrm{yrs}$ & $8 \mathrm{yrs}$ & $4 \mathrm{yrs}$ \\
\hline Age at evaluation & $20 \mathrm{yrs}$ & $15 \mathrm{yrs}$ & $11 \mathrm{yrs}$ \\
\hline BMI (Z-score) & 13th petl (-1.12) & $<1^{\text {st }} \operatorname{pctl}(-7.0)$ & $<1^{\text {st }}$ pctl $(-6.5)$ \\
\hline Back deformities & Severe scoliosis & Lordosis & Normal posture \\
\hline Foot deformities & Pes cavus & - & Pes cavus/varus \\
\hline Walking & Non-ambulatory & Steppage & Steppage \\
\hline Atrophy & Global, contractures & Global & Global \\
\hline Weakness & Generalized & Generalized & Generalized \\
\hline Reflexes & $\begin{array}{l}\text { Hyporeflexia, } \\
\text { Ach brisk }\end{array}$ & $\begin{array}{c}\text { Hyporeflexia, } \\
\text { Ach brisk }\end{array}$ & $\begin{array}{c}\text { Hyperreflexia, } \\
\text { Ach absent }\end{array}$ \\
\hline Tongue & $\begin{array}{l}\text { Wasted and } \\
\text { fasciculating }\end{array}$ & $\begin{array}{l}\text { Wasted and } \\
\text { fasciculating }\end{array}$ & $\begin{array}{l}\text { Wasted and } \\
\text { fasciculating }\end{array}$ \\
\hline Jaw jerk & Present & - & Present \\
\hline Respiratory & Trach. at $17 \mathrm{yrs}$ & - & Dyspnea on exercise \\
\hline Cognition & $\begin{array}{l}\text { Frontal lobe } \\
\text { dysfunction }\end{array}$ & Executive dysfunction & Normal \\
\hline Sensory & Normal & Normal & $\begin{array}{l}\text { Moderate pain loss in } \\
\text { glove-stocking, painless } \\
\text { foot ulceration }\end{array}$ \\
\hline \multicolumn{4}{|l|}{ Neurophysiology } \\
\hline Motor & Chronic denervation & $\begin{array}{c}\text { Acute and chronic } \\
\text { denervation }\end{array}$ & $\begin{array}{l}\text { Axonal loss } \\
\text { Polyphasia }\end{array}$ \\
\hline Sensory & Normal & Normal & Axonal loss \\
\hline Additional features & - & $\begin{array}{l}\text { Scapular winging, } \\
\text { Gower's sign }\end{array}$ & $\begin{array}{l}\text { Gower's sign, vitamin D } \\
\text { def., hyperhidrosis }\end{array}$ \\
\hline
\end{tabular}

*variant was detected in 1 of 49 and 0 of 149 next-generation sequencing reads from the father's saliva-derived and buccal-derived DNA. Ach, Achilles tendon; yrs, years; trach, tracheostomy; def., deficiency; pctl, percentile. 
Table 2. Demographic and clinical features of patients diagnosed with ALS carrying SPTLC1 mutations

Sample and mutation

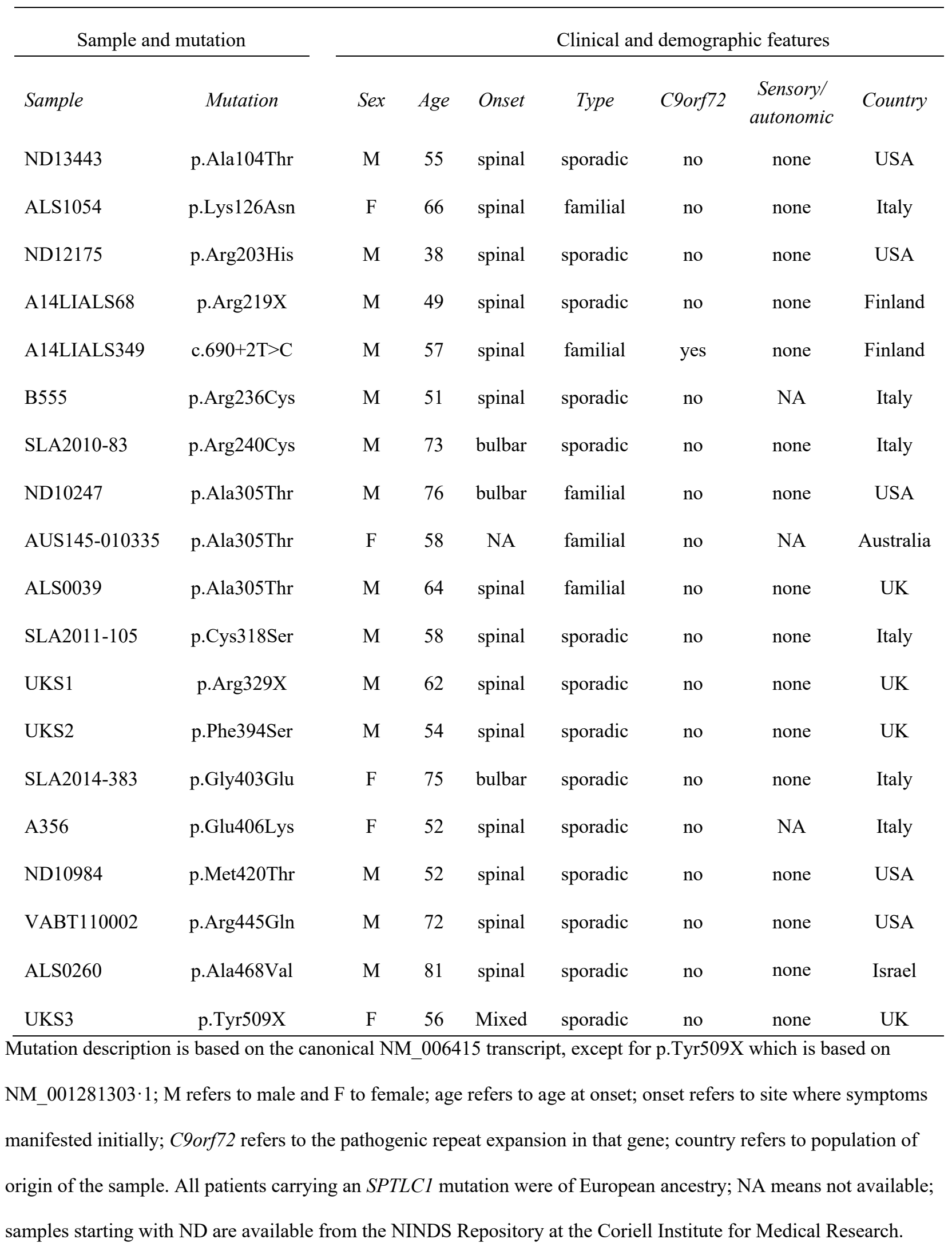

Clinical and demographic features

\footnotetext{
Mutation description is based on the canonical NM_006415 transcript, except for p.Tyr509X which is based on

NM_001281303·1; M refers to male and F to female; age refers to age at onset; onset refers to site where symptoms manifested initially; C9orf72 refers to the pathogenic repeat expansion in that gene; country refers to population of samples starting with ND are available from the NINDS Repository at the Coriell Institute for Medical Research.
} 